\title{
New experimental research stand SVICKA neutron field analysis using neutron activation detector technique
}

\author{
Jan Varmuza ${ }^{1, *}$, Karel Katovsky ${ }^{1}$ Miroslav Zeman ${ }^{1}$, Ondrej Stastny ${ }^{1}$, Ivan Haysak ${ }^{2}$ and \\ Robert Holomb ${ }^{1}$ \\ ${ }^{1}$ Brno University of Technology, FEEC, Technicka 12, 616 00, Brno, Czech Republic \\ ${ }^{2}$ Uzhhorod National University, Narodna Square 3, 88000, Uzhhorod, Ukraine
}

\begin{abstract}
Knowledge of neutron energy spectra is very important because neutrons with various energies have a different material impact or a biological tissue impact. This paper presents basic results of the neutron flux distribution inside the new experimental research stand SVICKA which is located at Brno University of Technology in Brno, Czech Republic. The experiment also focused on the investigation of the sandwich biological shielding quality that protects staff against radiation effects. The set of indium activation detectors was used to the investigation of neutron flux distribution. The results of the measurement provide basic information about the neutron flux distribution inside all irradiation channels and no damage or cracks are present in the experimental research stand biological shielding.
\end{abstract}

\section{Introduction}

In 2016 a new americium-beryllium neutron source was acquired at Department of electrical power engineering at Brno university of Technology. The main goal of the purchase was to provide better conditions for the basic research and the neutron energy spectrum analysis using the neutron activation detectors and measurement. The new measuring stand was designed for this purpose. The stand contains several irradiation channels and allows long term storing of the neutron source at the same time. The basic knowledge of the neutron flux distribution is required because of the further experiments and $\mathrm{R} \& \mathrm{D}$. The paper focuses on the measurement of neutron activation detectors and comparison of detector's response inside investigated irradiation channels of the irradiation stand. The basic characterization of thermal neutron spectra is performed using a cadmium spectral cover. The cadmium cover absorbs thermal neutrons in neutron spectra and calculated spectral index brings information about the ration between slow and fast plus epithermal neutrons.

\footnotetext{
* Corresponding author: varmuza@feec.vutbr.cz
} 


\section{Stand description}

The basic element of SVICKA (Stand with Various Irradiation Channels and Key Access) stand is composed of a high density polyethylene (HDPE) barrel full of paraffin. The second iron cask has the diameter $570 \mathrm{~mm}$. The icon cask is located inside the HDPE cask in its center.
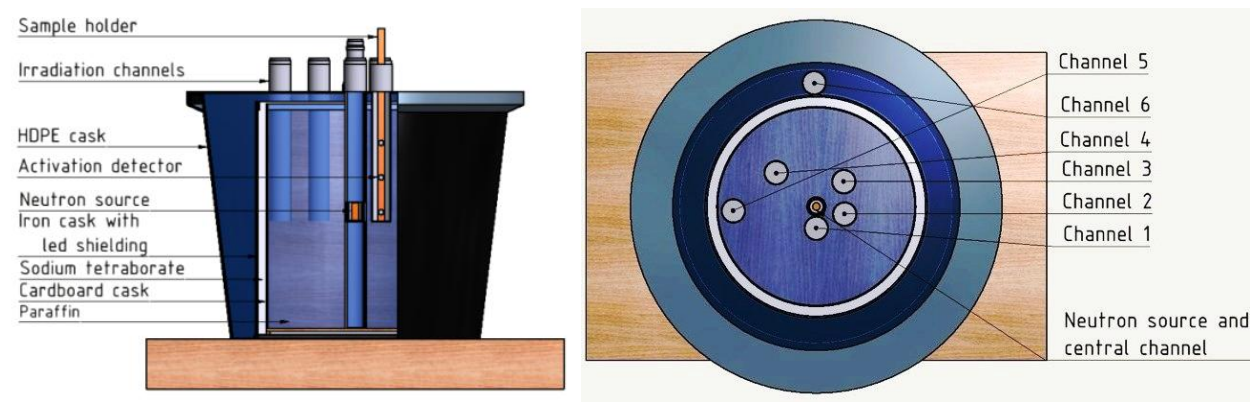

Fig. 1. The experimental irradiation stand.

The iron cask is covered with the $4 \mathrm{~mm}$ led layer that provides the basic gamma radiation shielding function. The next cardboard cask (the diameter $520 \mathrm{~mm}$ ) is in the center of iron cask. The gap between iron and cardboard casks is filled with a sodium tetraborate. The gab is $25 \mathrm{~mm}$ wide and the same thickness also has the sodium tetraborate layer on the top and on the bottom of iron cask. The sodium tetraborate provides neutron biological shielding function for slow neutrons.

There are 6 polyvinylchloride (PVC) tubes inside the cardboard cask that is also filled with the paraffin. These irradiation channels have $63 \mathrm{~mm}$ in the diameter and can be closed by removable plugs. The end of the plug is also filled with the sodium tetraborate because of the slow neutron leakage. The central channel is $50 \mathrm{~mm}$ in the diameter. There is the next removable tube with the diameter $36 \mathrm{~mm}$ containing the neutron source in the central channel. The removable tube also contains the removable plug full of paraffin and the end of the plug is filled with the sodium tetraborate.

The upper part of the stand also is covered with the $4 \mathrm{~mm}$ thick led layer. This layer provides gamma radiation shielding as well as a protection of paraffin and a working space. The central channel ends in the middle of the HDPE cask height. The channel 1 is located just next to the central channel and goes through the entire stand up to the cardboard cask bottom. Other channels end at the same vertical position like the neutron source. The channel 6 is located behind the sodium tetraborate layer and led shielding because of the low doses irradiation. Distances between central and irradiation channels are $0,2,4,8$, $16 \mathrm{~cm}$. The exception is the channel 6 . Its distance is $26.5 \mathrm{~cm}$ from the central channel because of inner shielding. 


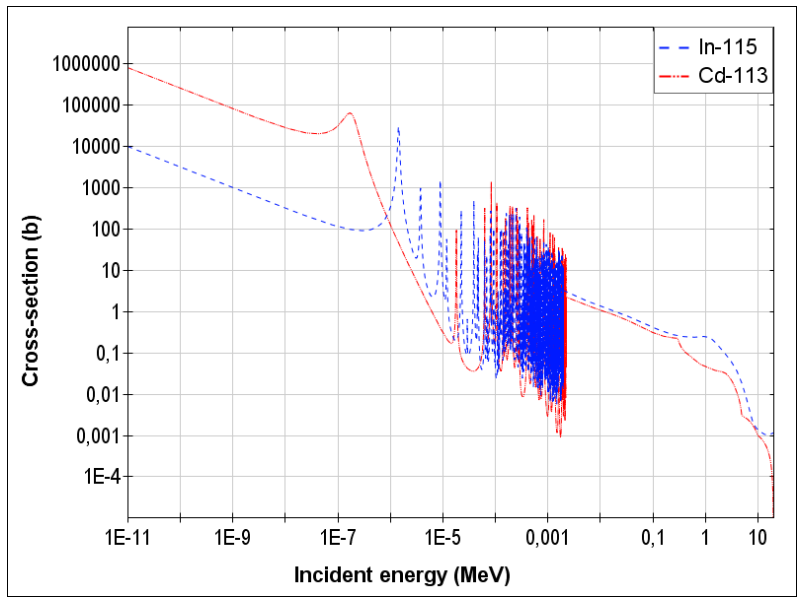

Fig. 2. Neutron cross section comparison [3].

\section{Experiment}

The neutron activation detectors and analysis were used for the basic analysis of irradiation channels neutron field and neutron energy spectra. Indium activation detectors with the diameter $16 \mathrm{~mm}$ and thickness $0.5 \mathrm{~mm}$ were used for irradiation in positions 0,10 and $20 \mathrm{~cm}$ above the neutron source. Then the second irradiation was carried out with the cadmium spectral cover in $0 \mathrm{~cm}$ vertical position. The fig. 2 shows the neutron capture cross section of Cd-113 and In-115.

The spectral index provides information about the ratio between slow and fast plus epithermal neutrons in the neutron spectrum. The spectral index can be calculated using measurement with the cadmium filter. The cadmium edge is approximately $0.5 \mathrm{eV}$ which is the value where the cross section falls before the local extreme [1]. That means neutrons with lower energy are strongly absorbed by the Cd-113. That means the response of the detector is caused by fast and epithermal neutrons while the detector was hidden inside the cover because all slow neutrons were captured in the cadmium cover. That is the reason why the comparison of the reaction rates with and without spectral cover provides the rough information about amount of slow neutrons in the spectra. All activation detectors were measured using the high purity germanium detector (HPGe).
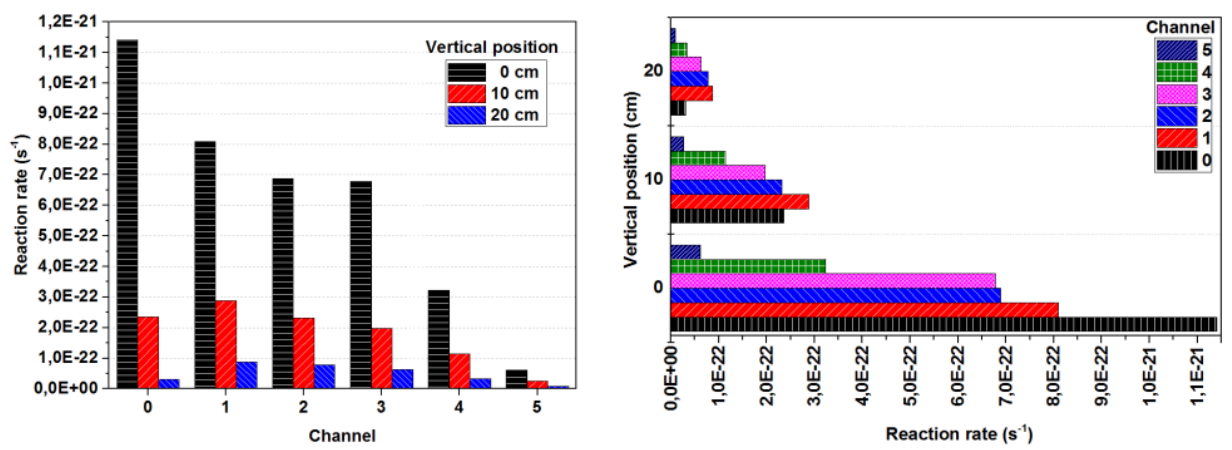

Fig. 3. Neutron flux distribution in irradiation channels and different height levels 


\section{Evaluation}

Reaction rates and spectral indices were calculated based on the indium detector measurement inside all channels and all height positions except of the channel 6 . The reaction rates and its distribution are shown in fig. 3 . The left part of fig. 3 shows the dependence of the reaction rate distribution inside irradiation channels and the distance from the neutron source.

The reaction rate of the detector depends mostly on the neutron flux, neutron energy spectra and material of the detector. The neutron flux is decreasing while the distance is increasing. When the neutron energy spectrum is not changing with increasing distance the reaction rate will also decrease. This can be seen in channels 3, 4 and 5. When the neutron energy spectrum is significantly changing between channels than the detector response is strongly affected by the neutron capture cross section. It can be seen in channels 2 and 3 where the decrease of the reaction rate is not significant but the difference in spectral index is significant. Reaction rates are almost the same in channels 2 and 3. That is related to diffuse length which is required for neutron slow down. There is just $2 \mathrm{~cm}$ of paraffin between central channel and channel 2. The paraffin diffuse length is longer than the distance between the neutron source and the irradiation channel [2]. That means the neutron spectrum is not fully thermalized. This can be seen in fig. 4 where reaction rates and spectral indices are calculated according formulas (1) and (2). In the fig. 4 the reason of the reaction rate increase is visible. The distance between channels 2 and 3 is greater than the diffuse length therefore the amount of thermal neutrons is higher than fast neutrons. These thermal neurons are absorbed in Cd filter and spectral index is higher. The reaction rate is relative to one target nucleus of irradiated material and one incident particle.

$$
\begin{gathered}
R_{R}=S\left(\mathrm{E}_{\gamma}\right) \cdot \frac{1}{N_{0}} \cdot \frac{1}{t_{\text {live }}} \cdot \frac{1}{1-e^{-\lambda \cdot t_{a}}} \cdot \frac{1}{e^{-\lambda \cdot t_{c}}} \cdot \frac{\lambda \cdot t_{\text {real }}}{1-e^{-\lambda \cdot t_{\text {real }}}} \cdot \frac{1}{I_{\gamma}\left(E_{\gamma}\right)} \cdot \frac{1}{\varepsilon_{F E P}^{\gamma}\left(E_{\gamma}\right)} \\
\text { spectral index }=\frac{R_{R}}{R_{R, C d}}
\end{gathered}
$$

Where $R_{R}$ is the reaction rate without cover, $S$ the peak area, $E_{\gamma}$ the gamma line energy, $\mathrm{N}_{0}$ the number of detector nuclei (calculated for each detector separately), $\mathrm{t}_{\text {live }}$ the live time of measurement, $t_{a}$ the irradiation time, $\lambda$ the decay constant, $t_{d}$ the cooling time between irradiation end and measurement start, $\mathrm{t}_{\text {real }}$ the real time of measurement, $\mathrm{I}_{\gamma}$ the gamma line intensity, $\varepsilon_{F E P}^{\gamma}$ the detector efficiency and $\mathrm{R}_{\mathrm{R}, \mathrm{Cd}}$ is the reaction rate with $\mathrm{Cd}$ cover.

The lower reaction rate is in the channel 5 because of the sodium tetraborate shielding that absorbs part of reflected neutrons. The fig. 3 also shows that the reaction rates are lower in the central channel than reaction rates in the channel 1 in vertical positions 10 and $20 \mathrm{~cm}$ above the neutron source. This is because of the activation detector mounting position. Activation detectors were located in the center of the central channel in the vertical position. This position caused that the self-shielding effect was present and total reaction rate was lower. 


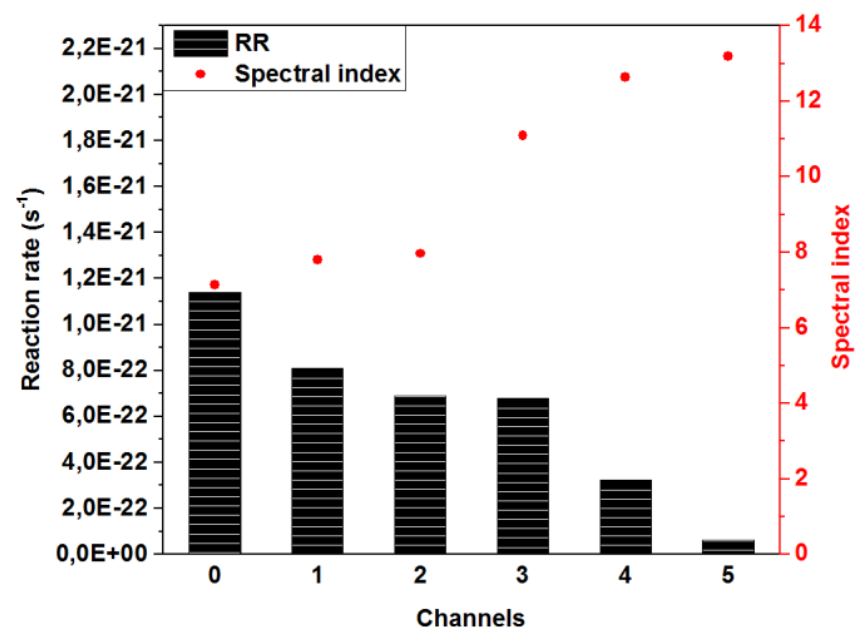

Fig. 4. Irradiation channel and spectral index comparison

The neutron energy characterization of carried out for the position $0 \mathrm{~cm}$ above the neutron source because of the highest detector response in all channels. The fig. 4 shows the change of ration between slow and fast + epithermal neutrons. The spectral index increases almost linearly. The lower value is in the last channel because part of slow neutrons is captured in shielding barrier. The drop signalizes lower presence of slow neutron in the channel 2 . A cavity could be responsible for harder neutron energy spectra between the neutron source and the channel 2. The other option is the relationship to the absorption cross section. The presence of the cavity has not very a high probability between the neutron source and the irradiation channel 2 therefore further investigation is required. The investigation will focus on the slighter changes of the moderator between the source and the activation detector.

\section{Conclusion}

The reaction rates were measured using neutron activation method and the analysis of the neutron flux distribution was done. The significant change of neutron energy spectra is shown in the fig. 4 in irradiation channels 2 and 3. The change of the reaction rate is very low even the distance between channels 2 and 3 is two times higher. This information is also supported by the spectral index calculation. The spectral index is almost $30 \%$ higher in the channel 3 than in the channel 2. Based on the measured data the significant decrease of the reaction rate is shown in the fig. 3 and fig. 4 in channel 5 because the sodium tetraborate shielding is present near this channel. The irradiation channels 2 and 3 are suitable for the further spectral filter experiment because the significant change of the neutron energy spectrum was observed.

This research work has been carried out in the Centre for Research and Utilization of Renewable Energy (CVVOZE). Authors gratefully acknowledge financial support from the Ministry of Education, Youth and Sports of the Czech Republic under NPU I programme (project No. LO1210), the Technology Agency of the Czech Republic (project No. TE01020455) and Joint Institute for Nuclear Research (project No. JINR2017_3a3_259_16). 


\section{References}

1. G.F.Knoll, Radiation detection and measurement: revue littéraire mensuelle. 4th ed. Hoboken, N.J.: John Wiley, 2010. ISBN 04-701-3148-9.

2. Y. San, Investigation of Neutron Diffusion Length in Wax Medium, Universities Research Journal 2011, Vol. 4, No. 4, [online] 2017 [cit. 2017-08-22] Available form: http://umoar.mu.edu.mm/bitstream/handle/123456789/192/URJ-Vol4-No4\%28\% 201D $\% 20 \% 20$ Heat $\% 20 \mathrm{Eq} \% 29$.pdf? sequence $=1 \&$ isAllowed $=\mathrm{y}$

3. Nuclear Energy Agency. Janis 4: Java-based Nuclear Data Information System [online] 2017. [cit. 2017-01-13]. Available form: http://www.oecd-nea.org/janis/ 